\title{
Discordant American Society of Anesthesiologists Physical Status Scoring between Anesthesiologists and Surgeons Is Correlated With Adverse Patient Outcomes: A Retrospective Cohort Study of 46284 Elective Surgical Patients
}

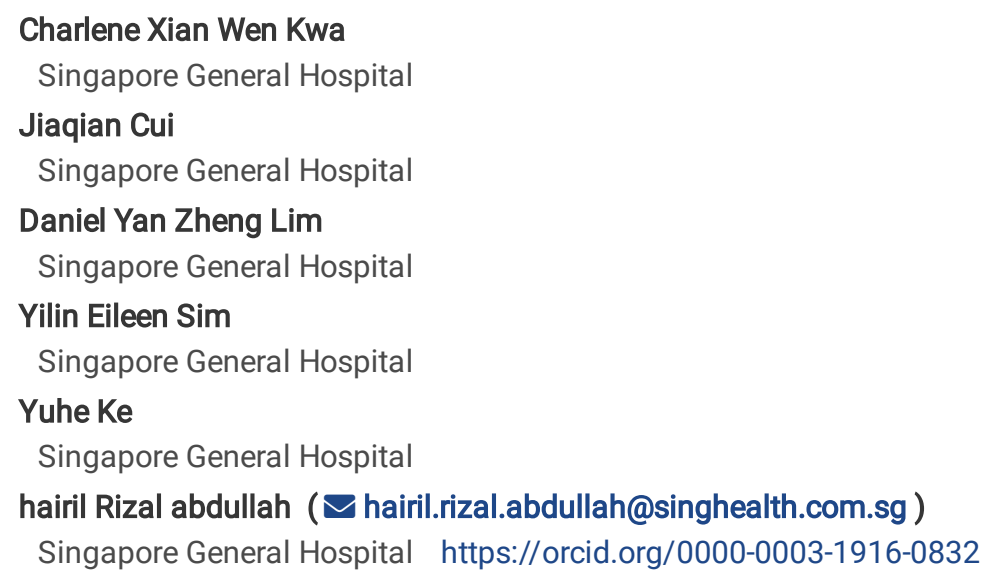




\section{Abstract}

\section{Background}

The American Society of Anesthesiologists Physical Status Classification (ASA) score is used for communication of patient health status, risk scoring, benchmarking and financial claims. Prior studies using hypothetical scenarios have shown poor concordance of ASA scoring among healthcare providers. However, there is a paucity of concordance studies using real-world data, as well as studies of clinical factors or patient outcomes associated with discordant scoring. The study aims to assess real-world ASA score concordance between surgeons and anesthesiologists, factors surrounding discordance and its impact on patient outcomes.

Methods

This retrospective cohort study was conducted in a tertiary academic medical center on 46284 consecutive patients undergoing elective surgery between January 2017 and December 2019. ASA scores entered by surgeons and anesthesiologists, patient demographics, and postoperative outcomes were collected. We assessed the concordance of preoperative ASA scoring between surgeons and anesthesiologists, clinical factors associated with score discordance, the impact of score discordance on clinically important outcomes, and the discriminative ability of the two scores for 30-day mortality, 1-year mortality, and intensive care unit (ICU) admission. Statistical tests used included Cohen's weighted $\mathrm{K}$ score, chi-square test, t-test, unadjusted odds ratios and logistic regression models.

Results

The ASA score showed moderate concordance (weighted Cohen's $K$ 0.53) between surgeons and anesthesiologists. 15098 patients (32.6\%) had discordant scores, of which 11985 (79.4\%) were scored lower by surgeons. We found significant associations between discordant scores and anesthesiologist-assessed comorbidities, patient age and race. Patients with discordant scores had a higher risk of 30-day mortality (odds ratio $2.00,95 \%$ confidence interval $[\mathrm{Cl}]=1.52-2.62, \mathrm{p}<0.0001$ ), 1 -year mortality (odds ratio $1.53,95 \% \mathrm{Cl}=1.38-1.69, \mathrm{p}<0.0001$ ), and ICU admission $>24$ hours (odds ratio $1.69,95 \% \mathrm{Cl}=1.47-1.94, \mathrm{p}<0.0001$ ), and stratified analyses showed a trend towards higher risk when the surgeons' ASA score was lower.

\section{Conclusions}

There is moderate concordance between surgeons and anesthesiologists in assigning the ASA classification. Discordant ASA scores are associated with adverse patient outcomes. Hence, there is a need for improved standardization of ASA scoring and cross-specialty review in ASA-discordant cases.

\section{Background}

The American Society of Anesthesiologists physical status classification (ASA) score is a widely utilized grading system first introduced in 1941 to assess and communicate the preoperative health of patients undergoing anesthesia.[1] It was revised in 1961,[2] and modified in 2014 to include clinical examples for each ASA score with the aim of improving inter-rater reliability or concordance. The modern ASA score consists of six categories ranging from Class 1 (describing a healthy patient) to Class 6 (referring to the brain-dead organ donor).[3]

ASA scoring has significance both clinically and from a health services perspective. While ASA scoring alone is not intended for the prediction of perioperative risks,[3] it has been shown to be independently predictive of perioperative morbidity and mortality [4] and is included as part of several perioperative risk assessment tools that are widely used by surgeons and anesthesiologists. These tools include the surgeons-authored National Surgical Quality Improvement Program risk calculator[5] and Gupta Myocardial Infarction or Cardiac Arrest calculator,[6] as well as the anesthesiologists-authored Surgical Assessment Risk Tool ${ }^{[7]}$ and Combined Assessment of Risk Encountered in Surgery. $[8,9]$ ASA scores are also frequently reported in healthcare benchmarking exercises and payer billing documentations.

Any significant discordance in ASA scoring between healthcare providers is therefore concerning and may subject patients to contradictory risk counseling and inappropriate perioperative plans. At a health system level, discordant ASA scoring may undermine efforts for quality assurance,[10] allocations of critical care resources, risk-based remuneration for health outcomes and may result in potential financial costs from over-scoring.[11]

Multiple studies have reported moderate to poor concordance of the ASA score among various clinicians.[12-17] In particular, one study observed a significant disagreement in ASA scoring between anesthesiologists and surgeons when presented with hypothetical patient scenarios and that surgeons consistently assigned lower scores.[18] However, there is a paucity of real-world data on this question as most real-patient studies of ASA concordance to date have been conducted among anesthesiologists[12-17] or were restricted to specific patient 
cohorts. $[19,20]$ This is an important evidentiary gap as both specialties jointly manage patients undergoing surgeries. Furthermore, the association between discordant ASA scores and adverse patient outcomes has not been comprehensively studied previously.

To fill these knowledge gaps, our study aims to examine the concordance of ASA scoring between surgeons scheduling patients for surgery and anesthesiologists conducting the outpatient preoperative evaluation. We further examined the clinical and demographic factors associated with discordant scoring and whether discordant scores were associated with adverse postoperative outcomes. Finally, we compared the discriminative ability of ASA scores assigned by surgeons and anesthesiologists in the prediction of postoperative outcomes.

\section{Methods}

\subsection{Study Design and Data Sources}

This was a single-center retrospective cohort study conducted in Singapore General Hospital, the largest tertiary academic medical center in Singapore. The local Institutional Review Board (CIRB Reference number 2020/2801) granted a waiver of consent due to the use of anonymized routinely collected clinical data and no patient interaction was required. The data analysis and statistical plan was written and filed with the Institutional Review Board before the data were accessed.

Our study cohort was extracted from the Perioperative and Anesthesia Subject Area, a curated electronic medical records database within our institution's enterprise data warehouse (SingHealth-IHiS Electronic Health Intelligence System) which contains the records of all operative procedures performed since 2015. The system integrates patient information such as patient demographics, laboratory results, comorbidities and postoperative outcomes from multiple healthcare transactional systems, such as the hospital's clinical information system (Sunrise Clinical Manager, Allscripts, Illinois, United States of America) and other administration and ancillary electronic systems. Mortality data on the system were synchronized with the National Electronic Health Records, including data from the National Registry of Births and Deaths, ensuring a near-complete mortality data follow-up.

In our institution, the ASA score is assigned by the surgeon on a standardized electronic admission form during the surgery listing process. Patients are then typically seen in the anesthesia preoperative clinic within a month of the surgery listing. Information on patient demographics, anthropometric parameters, preoperative comorbidities, and ASA score are routinely assessed by the attending anesthesiologist as part of structured clinical notes during the pre-operative assessment, and are included within the database. The 2014 ASA scoring definition along with their full published examples are available for reference in the clinic. While the anesthesiologist can potentially access the surgeon's ASA score, it is usually independently assigned in our center. There are no financial incentives in assigning higher ASA scores both for anesthesiologists and surgeons within our local healthcare system.

\subsection{Participant Cohort and Variables}

We included all patients aged 18 years old and above undergoing elective surgery under general or regional anesthesia or monitored anesthesia care between January 2017 to December 2019. Patients who underwent cardiac surgery, transplant surgery, or surgery for burns injuries were excluded. Patients planned for elective cardiac surgery in our center undergo preoperative anesthesia screening by the surgeon who fills in the pre-anesthesia assessment form before the patient is assessed by the anesthesiologist, while patients requiring transplant surgery would usually have a standardized ASA score as there is organ failure necessitating the surgery. Patients with a missing ASA score by either the surgeon or anesthesiologist and patients assigned an ASA score of 5 or 6 by either the surgeon or anesthesiologist were also excluded (Fig. 1).

For each patient, we obtained preoperative data such as age, sex, race, surgical specialty, and comorbidities including ischemic heart disease, congestive heart failure, cerebrovascular accidents, diabetes mellitus requiring insulin, and hypertension. These comorbidities are assessed by the anesthesiologist as part of the Revised Cardiac Risk Index, which is routinely used in our institution.[21] The ASA scores assigned by both the anesthesiologist and surgeon were obtained, and the relevant clinical outcomes (death within 30 days, death within 1 year, ICU admission for $>24$ hours) were determined.

Additional File 1(Supplemental Table 1) compares the characteristics of 264 patients who were excluded from our study as they had no valid ASA scores. All 264 patients had missing anesthesiologist ASA scores and there was no statistically significant difference between patients in the final cohort and the excluded patients for demographic variables (age, sex, race) and clinical outcomes. Fewer of the excluded patients had anesthesiologist-assessed comorbidities, and the differences were statistically significant for some. Our interpretation is that patients with incomplete anesthesiologist ASA scores are more likely to have other areas incompletely assessed by the anesthesiologist. Overall, the number of such patients is small and not deemed to be a major source of bias.

\subsection{Statistical Analysis}

Page 3/12 
Analyses were performed using Python version 3.7.1 and R version 4.0.2 with their base utility functions. Additional packages used in $\mathrm{R}$ included the "questionr" package for multivariate logistic regression, "pROC" for receiver operating characteristic curve analyses, and "irr" for concordance analyses.

\subsubsection{Assessment of Agreement between Surgeon and Anesthesiologist ASA Scores}

Cross tabulation was performed for the anesthesiologist's ASA score against the surgeon's ASA score. Concordance between these two variables was determined using Cohen's weighted $K$. The $K$-statistic was interpreted in the manner of Landis and Koch as slight $(0-0.2)$, fair (0.21-0.4), moderate (0.41-0.6), substantial (0.61-0.8) and almost perfect (0.81-1.0) agreement.[22]

Our sample was drawn from a database that exhaustively documents all surgeries performed within the hospital, and we considered all sequential patients within the study time frame (January 2017 to December 2019). As a comparison, the sample size calculation to detect a moderate agreement $(K>0.4)$ and exclude a fair agreement $(K=0.2)$ with a one-sided $95 \%$ confidence interval and $90 \%$ power is 186 .

\subsubsection{Descriptive Statistics for Overall Cohort and Subgroup Analyses of Discordant ASA Scores}

Descriptive statistics were calculated and expressed as counts and percentages for categorical data, and means with standard deviation for continuous data. The cohort was stratified into patients with concordant and discordant ASA scores. Univariate statistical analysis was performed using the chi-square test for categorical variables and the t-test for continuous variables. Subgroup analyses were also performed comparing patients where the surgeon assigned a lower ASA score against patients with a concordant ASA score, and likewise comparing patients where the anesthesiologist assigned a lower ASA score against patients with a concordant ASA score. In view of the multiple statistical comparisons, Bonferroni's correction was used and the $p$-value cut-off for statistical significance was determined to be $p<0.001$.

\subsubsection{Effect of Discordant ASA Scores on Clinical Outcomes}

The discordance of ASA scores between surgeons and anesthesiologists was calculated and stratified in several different ways. Three forms to express discordance were used. Firstly, as a binary variable representing whether the ASA scores were discordant or not; secondly, as a ternary variable representing whether the ASA scores were concordant, surgeon ASA score was lower, or anesthesiologist ASA score was lower; and lastly, as the raw difference with appropriate binning of categories with low counts. These variables, representing different ways of stratifying the degree of ASA score discordance, were separately entered as the sole predictive variable into logistic regression models. A separate model was fitted for each of the clinical outcomes of death within 30 days, death within 1 year, and ICU admission for $>24$ hours. The unadjusted odds ratios and p-values were calculated for each stratum of ASA discordance, with the ASA concordant patients as the reference group.

\subsubsection{Comparison of Surgeon and Anesthesiologist ASA Score Discriminative Ability}

Logistic regression models using the ASA score as a sole predictor were fitted for surgeon and anesthesiologist ASA scores for the outcomes of 30-day mortality, 1-year mortality, and ICU admission $>24$ hours. The receiver operating characteristic curve, area under the receiver operating characteristic curve (AUC), and its $95 \%$ confidence interval were used to determine each model's discriminative ability. DeLong's method was used to compare for statistically significant differences between the receiver operating characteristic curves of models based on anesthesiologist ASA scores versus those based on surgeon ASA scores.[23]

\section{Results}

\subsection{Concordance of Surgeon and Anesthesiologist ASA scores}

Our final study cohort comprised 46284 patients, of which $46.4 \%$ (21474/46284) were male and 53.6\% (24810/46284) were female. The cross-tabulation of surgeon and anesthesiologist ASA scores for all cases is presented in Table 1. The weighted Cohen's $K$ for concordance between surgeon and anesthesiologist scores was 0.53 , signifying moderate agreement. 


\begin{tabular}{|llllll|}
\hline & \multicolumn{5}{c|}{ ASA score by Surgeon } \\
\cline { 2 - 6 } ASA score by Anesthesiologist & $\mathbf{1}$ & $\mathbf{2}$ & $\mathbf{3}$ & $\mathbf{4}$ \\
\cline { 2 - 6 } & $\mathbf{1}$ & 4247 & 2160 & 7 & 0 \\
\hline $\mathbf{2}$ & 5014 & 22996 & 867 & 8 \\
\hline $\mathbf{3}$ & 285 & 6364 & 3869 & 71 \\
\hline 4 & 1 & 86 & 235 & 74 \\
\hline
\end{tabular}

\subsection{Descriptive statistics and stratified analyses}

$67.4 \%$ of patients (31186/46284) had a concordant ASA score given by both surgeons and anesthesiologists. Descriptive statistics for the ASA concordant and discordant groups are presented in Table 2. $79.4 \%$ of patients with discordant scores (11985/15098) had a lower ASA score assigned by the surgeon, and 20.6\% (3113/15098) had a lower ASA score assigned by the anesthesiologist.

For all baseline patient characteristics, there were significant differences between patients with concordant and discordant scores, with exception of the male sex and the presence of raised creatinine. Discordant ASA scores overall were associated with a higher risk of all adverse outcomes- death at 30 days, death at 1 year, and ICU admission of more than 24 hours. When the discordant ASA scores were further stratified, we observed that a lower surgeon ASA score was associated with all negative outcomes. The magnitude of risk was greater the lower the surgeon ASA score was compared to the anesthesiologist. On the other hand, a lower anesthesiologist ASA score was only associated with ICU admission $>24$ hours but not death at 30 days or 1 year. This is depicted in Fig. 2, with additional details included in Additional file 2 (Supplemental Table 2).

The AUC of 30-day mortality for ASA score assigned by anesthesiologists was 0.824 ( $95 \% \mathrm{Cl} 0.799-0.849$ ) and for ASA score assigned by surgeons was $0.756(95 \% \mathrm{Cl} 0.728-0.784)(p<0.001$ for difference) (Fig. 3A). For 1-year mortality, the AUC for ASA score assigned by anesthesiologists was $0.765(95 \% \mathrm{Cl} 0.755-0.775)$ and $0.712(95 \% \mathrm{Cl} 0.702-0.723)$ for the ASA score assigned by surgeons ( $<<0.001$ for difference) (Fig. 3B). For ICU admission $>24$ hours, the AUC for ASA score assigned by anesthesiologists and surgeon were $0.782(95 \% \mathrm{Cl}$ $0.767-0.797)$ and 0.767 ( $95 \% \mathrm{Cl} 0.751-0.783$ ) respectively ( $p=0.060$ for difference) (Fig. 3C).

Table 2: Baseline patient characteristics stratified by the concordance of ASA scores given by surgeons and anesthesiologists 


\begin{tabular}{|c|c|c|c|c|c|c|c|c|c|}
\hline & & $\begin{array}{l}\text { Overall } \\
{[n=} \\
46284]\end{array}$ & $\begin{array}{l}\text { Concordant } \\
\text { ASA Score } \\
{[n=31186]}\end{array}$ & $\begin{array}{l}\text { Discordant } \\
\text { ASA Score } \\
\text { [n=15098] }\end{array}$ & $\begin{array}{l}\text { p- } \\
\text { value }^{a}\end{array}$ & $\begin{array}{l}\text { Surgeon } \\
\text { ASA } \\
\text { scored } \\
\text { lower } \\
\text { [n= } \\
11985]\end{array}$ & $\begin{array}{l}\text { p- } \\
\text { value }^{b}\end{array}$ & $\begin{array}{l}\text { Anesthesiologist } \\
\text { ASA scored } \\
\text { lower }[n=3113]\end{array}$ & p-value ${ }^{c}$ \\
\hline \multicolumn{2}{|c|}{ Male sex, No.(\%) } & $\begin{array}{l}21474 \\
(46.4)\end{array}$ & $\begin{array}{l}14312 \\
(46.0)\end{array}$ & $\begin{array}{l}7162 \\
(47.4)\end{array}$ & 0.0002 & $\begin{array}{l}5670 \\
(47.3)\end{array}$ & 0.0072 & $1492(47.9)$ & 0.031 \\
\hline \multicolumn{2}{|c|}{$\begin{array}{l}\text { Age (Time of Surgery), mean } \\
\text { (SD) }\end{array}$} & $\begin{array}{l}58.0 \\
(16.0)\end{array}$ & $59.0(16.0)$ & $56.0(17.0)$ & $\begin{array}{l}<.0001 \\
0 .\end{array}$ & $\begin{array}{l}56.0 \\
(17.0)\end{array}$ & $\dot{0} 0001$ & $54.0(16.0)$ & <. \\
\hline \multirow[t]{4}{*}{$\begin{array}{l}\text { Race, No. } \\
(\%)\end{array}$} & Chinese & $\begin{array}{l}34560 \\
(74.7)\end{array}$ & $\begin{array}{l}23814 \\
(76.4)\end{array}$ & $\begin{array}{l}10746 \\
(71.2)\end{array}$ & \multirow[t]{4}{*}{$<_{0.0001}$} & $\begin{array}{l}8463 \\
(70.6)\end{array}$ & \multirow[t]{4}{*}{$<.0001$} & $2283(73.3)$ & \multirow[t]{4}{*}{$\dot{0}_{0.0001}$} \\
\hline & Indian & $\begin{array}{l}4459 \\
(9.6)\end{array}$ & $2793(9.0)$ & $\begin{array}{l}1666 \\
(11.0)\end{array}$ & & $\begin{array}{l}1336 \\
(11.2)\end{array}$ & & $330(10.6)$ & \\
\hline & Malay & $\begin{array}{l}4111 \\
(8.9)\end{array}$ & $2558(8.2)$ & $\begin{array}{l}1553 \\
(10.3)\end{array}$ & & $\begin{array}{l}1306 \\
(10.9)\end{array}$ & & $247(7.9)$ & \\
\hline & Others & $\begin{array}{l}3154 \\
(6.8)\end{array}$ & $2021(6.5)$ & $1133(7.5)$ & & $\begin{array}{l}880 \\
(7.3)\end{array}$ & & $253(8.1)$ & \\
\hline \multicolumn{2}{|c|}{ Creatinine > 2mg/dl, No.(\%) } & $\begin{array}{l}2281 \\
(4.9)\end{array}$ & $1507(4.8)$ & $774(5.1)$ & 0.18 & $\begin{array}{l}722 \\
(6.0)\end{array}$ & $\begin{array}{l}< \\
0.0001\end{array}$ & $52(1.7)$ & $<0.0001$ \\
\hline \multicolumn{2}{|c|}{$\begin{array}{l}\text { Diabetes mellitus on Insulin, No. } \\
(\%)\end{array}$} & $\begin{array}{l}1750 \\
(3.8)\end{array}$ & $1025(3.3)$ & $725(4.8)$ & $\dot{0.0001}$ & $\begin{array}{l}681 \\
(5.7)\end{array}$ & $\dot{0.0001}$ & $44(1.4)$ & $<.0001$ \\
\hline \multicolumn{2}{|c|}{$\begin{array}{l}\text { History of Congestive heart } \\
\text { failure, No.(\%) }\end{array}$} & $\begin{array}{l}1065 \\
(2.3)\end{array}$ & $608(2.0)$ & $457(3.0)$ & $\begin{array}{l}<.0001 \\
0.00\end{array}$ & $\begin{array}{l}432 \\
(3.6)\end{array}$ & $\dot{0} 0001$ & $25(0.8)$ & $<$. \\
\hline \multicolumn{2}{|c|}{$\begin{array}{l}\text { History of Cerebrovascular } \\
\text { accident, No.(\%) }\end{array}$} & $\begin{array}{l}1529 \\
(3.3)\end{array}$ & $799(2.7)$ & $730(4.8)$ & $\dot{0} 0001$ & $\begin{array}{l}683 \\
(5.7)\end{array}$ & $\dot{0} 0001$ & $47(1.5)$ & 0.00039 \\
\hline \multicolumn{2}{|c|}{$\begin{array}{l}\text { History of Ischemic heart } \\
\text { disease, No.(\%) }\end{array}$} & $\begin{array}{l}4635 \\
(10.0)\end{array}$ & $2500(8.0)$ & $\begin{array}{l}2135 \\
(14.1)\end{array}$ & $\dot{0.0001}$ & $\begin{array}{l}1970 \\
(16.4)\end{array}$ & $\dot{0} 0001$ & $165(5.3)$ & $<.0001$ \\
\hline \multicolumn{2}{|c|}{ History of Hypertension, No.(\%) } & $\begin{array}{l}19225 \\
(41.5)\end{array}$ & $\begin{array}{l}13591 \\
(43.6)\end{array}$ & $\begin{array}{l}5634 \\
(37.3)\end{array}$ & $\begin{array}{l}<.0001 \\
0.0\end{array}$ & $\begin{array}{l}5070 \\
(42.3)\end{array}$ & 0.021 & $564(18.1)$ & $\begin{array}{l}< \\
0.0001\end{array}$ \\
\hline \multicolumn{2}{|c|}{ History of Smoking, No.(\%) } & $\begin{array}{l}4327 \\
(9.4)\end{array}$ & $2672(8.6)$ & $\begin{array}{l}1655 \\
(11.0)\end{array}$ & $\begin{array}{l}< \\
0.0001\end{array}$ & $\begin{array}{l}1434 \\
(12.0)\end{array}$ & $\begin{array}{l}< \\
0.0001\end{array}$ & $221(7.1)$ & 0.0055 \\
\hline \multirow{7}{*}{$\begin{array}{l}\text { Surgical } \\
\text { Specialty, } \\
\text { No.(\%) }\end{array}$} & Orthopedics & $\begin{array}{l}14523 \\
(31.4)\end{array}$ & $\begin{array}{l}10472 \\
(33.6)\end{array}$ & $\begin{array}{l}4051 \\
(26.8)\end{array}$ & \multirow[t]{7}{*}{$\begin{array}{l}<.0001 \\
0.00\end{array}$} & $\begin{array}{l}2958 \\
(24.7)\end{array}$ & \multirow[t]{7}{*}{$\begin{array}{l}<.0001 \\
0 .\end{array}$} & $1093(35.1)$ & \multirow[t]{8}{*}{$<.0001$} \\
\hline & General Surgery & $\begin{array}{l}11294 \\
(24.4)\end{array}$ & $7501(24.1)$ & $\begin{array}{l}3793 \\
(25.1)\end{array}$ & & $\begin{array}{l}3062 \\
(25.6)\end{array}$ & & $731(23.5)$ & \\
\hline & Urology & $\begin{array}{l}6403 \\
(13.8)\end{array}$ & $4472(14.3)$ & $\begin{array}{l}1931 \\
(12.8)\end{array}$ & & $\begin{array}{l}1503 \\
(12.5)\end{array}$ & & $428(13.8)$ & \\
\hline & $\begin{array}{l}\text { Obstetrics and } \\
\text { Gynecology }\end{array}$ & $\begin{array}{l}4799 \\
(10.4)\end{array}$ & $2803(9.0)$ & $\begin{array}{l}1996 \\
(13.2)\end{array}$ & & $\begin{array}{l}1826 \\
(15.2)\end{array}$ & & $170(5.7)$ & \\
\hline & Otorhinolaryngology & $\begin{array}{l}2844 \\
(6.1)\end{array}$ & $1817(5.8)$ & $1027(6.8)$ & & $\begin{array}{l}876 \\
(7.3)\end{array}$ & & $151(4.9)$ & \\
\hline & Vascular & $\begin{array}{l}2146 \\
(4.6)\end{array}$ & $1418(4.6)$ & $728(4.8)$ & & $\begin{array}{l}582 \\
(4.9)\end{array}$ & & $146(4.7)$ & \\
\hline & Plastics & $\begin{array}{l}1468 \\
(3.2)\end{array}$ & $942(3.0)$ & $526(3.5)$ & & $\begin{array}{l}388 \\
(3.2)\end{array}$ & & $138(4.4)$ & \\
\hline \multicolumn{9}{|c|}{ a $\mathrm{p}$-value for difference between ASA concordant and discordant patients } & \\
\hline \multicolumn{10}{|c|}{${ }^{b} p$-value for difference between ASA concordant patients and those scored lower by the surgeon } \\
\hline
\end{tabular}




\begin{tabular}{|c|c|c|c|c|c|c|c|c|}
\hline & $\begin{array}{l}\text { Overall } \\
{[n=} \\
46284]\end{array}$ & $\begin{array}{l}\text { Concordant } \\
\text { ASA Score } \\
\text { [n=31186] }\end{array}$ & $\begin{array}{l}\text { Discordant } \\
\text { ASA Score } \\
\text { [n=15098] }\end{array}$ & $\begin{array}{l}\text { p- } \\
\text { value }^{a}\end{array}$ & $\begin{array}{l}\text { Surgeon } \\
\text { ASA } \\
\text { scored } \\
\text { lower } \\
\text { [n }= \\
11985]\end{array}$ & $\begin{array}{l}\text { p- } \\
\text { value }^{b}\end{array}$ & $\begin{array}{l}\text { Anesthesiologist } \\
\text { ASA scored } \\
\text { lower }[n=3113]\end{array}$ & p-value ${ }^{c}$ \\
\hline Neurosurgery & $\begin{array}{l}730 \\
(1.6)\end{array}$ & $399(1.3)$ & $331(2.2)$ & & $\begin{array}{l}231 \\
(1.9)\end{array}$ & & $100(3.2)$ & \\
\hline Others & $\begin{array}{l}2077 \\
(4.5)\end{array}$ & $1362(4.4)$ & $715(4.7)$ & & $\begin{array}{l}559 \\
(4.7)\end{array}$ & & $156(5.01)$ & \\
\hline \multicolumn{9}{|c|}{ a $\mathrm{p}$-value for difference between ASA concordant and discordant patients } \\
\hline \multicolumn{9}{|c|}{ b p-value for difference between ASA concordant patients and those scored lower by the surgeon } \\
\hline
\end{tabular}

\section{Discussion}

\subsection{General Discussion}

Our results provide real-world evidence of differences in ASA scoring between surgeons and anesthesiologists after the 2014 ASA score modification, which have previously been studied only in hypothetical scenarios [18, 24] or between anesthesiologists and Internal Medicine providers. [25] Furthermore, we found that discordant ASA scores are associated with adverse outcomes, particularly when the surgeonassigned ASA score is lower.

The observed moderate concordance $(\kappa$ 0.53) in our study is consistent with that reported in the retrospective cohort study by Sankar et al between anesthesiologists in the preoperative clinic and on the day of surgery ( $K$ value 0.61 ) before the 2014 ASA score modification.[26] Another real-world study by Abouleish et al of concordance between anesthesiologists in the preoperative clinic and on the day of surgery had similar results ( $K$ value 0.62$)$, but subsequently demonstrated 'almost perfect' agreement ( $K$ value 0.85$)$ after the introduction of examples that were ASA and institutionally approved.[27]

The majority of the discordant scores were scored lower by surgeons, with the largest group comprising those assigned ASA 2 by the anesthesiologist but ASA 1 by the surgeon. We observed that patients with discordant ASA scores had a significantly higher proportion of comorbid clinical conditions (raised creatinine, diabetes mellitus on insulin, history of congestive heart failure, cerebrovascular accident, ischemic heart disease and smoking). This reflects the continuing subjectivity of the ASA scoring system despite the 2014 update, which was intended to improve concordance. The differences in recognition and perceived significance of comorbidities are likely to be a major contributing factor to the discordant ASA scores.

As the ASA score is a component of several major surgical risk scoring systems used by both surgeons and anesthesiologists in clinical care, discordant ASA scores can adversely impact the reliability of perioperative risk scoring and subsequent risk counseling. The ASA score is routinely used in deciding what pre-operative tests a patient requires at our institution and in other countries such as the United Kingdom. [28] Overestimation of the ASA score would increase the number of investigations a patient has before surgery, incurring unnecessary financial costs to the patient and healthcare system, while an underestimation of the ASA score may compromise patient safety. At the health systems level, discordant scores also can affect the allocation of critical care resources and undermine the use of the score in healthcare reimbursement and quality assurance efforts. This may disadvantage healthcare institutions financially and in inter-institutional rankings depending on which score is being reported to the external agencies. Other studies have shown that the addition of examples to the ASA score and reinforcement of its use were required to improve reliability. [27, 29] Standardization efforts are needed to improve the utility of ASA scores in clinical practice and for uses beyond the original intention of communicating patient healthcare status.

We also note that certain demographic factors were associated with discordant ASA scores, such as in younger patients and those of minority ethnicity. We postulate that younger patients may be perceived to have lower severity of disease by some clinicians, hence grading them with a lower score. Minority race patients may face communication or cultural barriers in disease and symptom communication and this may adversely affect accurate healthcare assessment. Ideally, demographic factors should not influence ASA scoring, which should be 
an objective reflection of patient physical status. This finding further supports the need for better standardization and education on ASA scoring.

Our study revealed that patients with discordant ASA scores had poorer clinical outcomes. With respect to mortality, stratified analyses of discordant ASA scores showed that patients whose surgeon assigned a lower score had a higher risk of 30-day and 1-year mortality. The lower the surgeon ASA score when compared to the anesthesiologist ASA score, the higher the risk was for 30-day and 1-year mortality. In contrast, patients with discordant ASA who were scored lower by their anesthesiologist did not have such an association. This is noteworthy, given that simple differences in medical opinion leading to discordant patient assessments would not ordinarily be expected to correlate with patient outcomes. Considering our analysis of ASA score discriminative ability, where anesthesiologists ASA scores had better discriminative ability for 30-day and 1-year mortality compared to those assigned by surgeons, this suggests that under-recognition of comorbidities by the surgeons might have resulted in an inaccurately optimistic ASA scoring in the discordant cases. Failure to recognize a high perioperative risk patient or interval development of comorbidity in the short timespan between surgeon and anesthesiologist review could have contributed to the poorer patient outcomes seen in this group.

On the other hand, all ASA discordant patients had a higher risk of ICU admission > 24 hours, in overall and stratified analyses. There was no significant difference in the discriminative ability between surgeon or anesthesiologist ASA scores for ICU admission $>24$ hours. This could possibly reflect differences in opinion being resolved at the point of surgery in favor of the more conservative decision to admit the patient post-operatively to ICU.

\subsection{Study strengths and limitations}

Our study's main strengths are that it was conducted in a large patient cohort spanning multiple years and encompassing the major categories of elective noncardiac surgery. Data collected was from 2017 onwards, after the 2014 ASA score revision and with adequate timelapse for familiarization, and hence does not span periods with potentially different interpretations of the score. The data used was derived from clinical databases, rather than administrative or financial records. Furthermore, as neither surgeons nor anesthesiologists have financial incentives tied to ASA scoring at our institution. This eliminates an important source of bias as its presence has been shown to be associated with potential upcoding of the ASA score.[30]

A limitation of our study is that the assignment of ASA score by surgeons and anesthesiologists for each patient was not done simultaneously. At our institution, surgeons assign the ASA score when listing the patient for surgery and anesthesiologists assign their score after that at the pre-operative assessment. As such, while the surgeon is completely blinded to the anesthesiologist's score, the anesthesiologist could be aware of the surgeon's score. However, our anesthesiologists generally make an independent assessment of the patient's healthcare status. The anesthesiologist assessment is also closer to the day of surgery than the surgeon's and hence the anesthesiologists' score has better recency. It is also possible that the patient's health could have deteriorated in the period of time between the surgeon and anesthesiologist review, accounting for score discordance and association with poorer outcomes. However, the waiting time for pre-operative assessment at our institution is generally short and most elective surgeries are premised on a relatively stable patient physical status. We do not deem this to be a major source of bias.

As near- contemporaneous ASA scoring was mandatory for both anesthesiologist and surgeon during the study period, potential sources of bias (e.g. recall bias, selection bias) that may affect retrospective studies are much less likely in our study. There was a very small proportion of potential patients (264 patients, $<1 \%$ ) who had missing anesthesiologist ASA scores. However, as addressed in Additional file 1 , this is unlikely to be a major source of bias.

As our study only included patients who underwent elective surgery, its outcomes should not be generalized to emergency cases. Cardiac, burns, and transplant surgery patients were also excluded, and our results may not apply in these groups of patients. Finally, as this was a single center study, this may limit generalizability, particularly in centers where ASA scores impact financial reimbursements (which is not present in our center) or centers with significantly different care patterns or patient comorbidity profiles.

\subsection{Opportunities for future work}

Our study data did not contain information that could individually identify the anesthesiologists or the surgeons assigning ASA scores. As such, we were unable to control for clinician factors that might have influenced the accuracy of the ASA score, such as level of training and seniority. Our information about comorbidities assessed by the clinicians, which directly impacts the ASA score, was limited to the anesthesiologists only (as there was no standardized assessment form for surgeon-assessed comorbidities during the period of study). Future analyse of ASA discordance may investigate these aspects further, to better understand the mechanisms of ASA discordance and other possible factors that influence it. 
The association of discordant ASA scores with adverse patient outcomes is a cause for concern. Besides further education and reinforcement of standard ASA examples, there may be a need for quality improvement studies to determine if specific conditions require more detailed or contextualized examples within the institution. Discordant ASA scores may be a red flag for missed comorbidities or interval development of new comorbidities, and mandatory cross-specialty review in ASA discordant cases is a potential intervention to ensure that patients are accurately assessed and appropriately prepared for surgery.

\section{Conclusion}

In a large single-center cohort study that was performed after the 2014 revision of the ASA score, there was moderate concordance between ASA scores assigned by anesthesiologists and surgeons in patients undergoing elective surgery. The majority of discordant patients were assigned a lower score by surgeons and is likely due to differences in recognition and grading of comorbidities. Patients with discordant ASA scores, and in particular those assigned lower ASA scores by surgeons, had a higher likelihood of 30-day mortality, 1-year mortality, and ICU admission > 24 hours. Our results suggest a need for improvement in the standardization of ASA scoring and that discordant ASA assessments may be a red flag for missed comorbidities.

\section{Abbreviations}

ASA

American Society of Anesthesiologists

ICU

Intensive care unit

$\mathrm{Cl}$

Confidence interval

AUC

Area under the receiver operating characteristic curve

\section{Declarations}

Ethics approval and consent to participate

The local Institutional Review Board (CIRB Reference number 2020/2801) granted a waiver of consent due to the use of anonymized routinely collected clinical data and no patient interaction was required. The data analysis and statistical plan was written and filed with the Institutional Review Board before the data were accessed.

\section{Consent for publication}

Not applicable.

\section{Competing interests}

The authors declare that they have no competing interests.

\section{Funding}

No funding was required in the research.

\section{Authors' contributions}

CXWK, principal investigator: Study design, interpretation of results, writing of manuscript. JC: interpretation of results, writing of manuscript. DYZL: data collection and analysis, interpretation of results, writing of manuscript. YES: editing of manuscript. YK: editing of manuscript. HRA: Research idea, study design, editing of manuscript, supervision.

\section{Acknowledgements}

Not applicable. 
The datasets used and/or analyzed during the current study are available from the corresponding author on reasonable request.

\section{References}

1. Saklad M. Grading of patients for surgical procedures. Anesthesiology. 1941;2:281-4. https://doi.org/10.1097/00000542-19410500000004.

2. Dripps RD, Lamont A, Eckenhoff JE. The Role of Anesthesia in Surgical Mortality. JAMA. 1961;178:261-6. https://doi.org/10.1001/jama.1961.03040420001001.

3. ASA Physical Status Classification System. American Society of Anesthesiologists 2020. https://www.asahq.org/standards-andguidelines/asa-physical-status-classification-system (accessed April 2021).

4. Hackett NJ, De Oliveira GS, Jain UK, Kim JYS. ASA class is a reliable independent predictor of medical complications and mortality following surgery. Int J Surg. 2015;18:184-90. https://doi.org/10.1016/j.ijsu.2015.04.079.

5. Davenport DL, Bowe EA, Henderson WG, Khuri SF, Mentzer RM. National Surgical Quality Improvement Program (NSQIP) Risk Factors Can Be Used to Validate American Society of Anesthesiologists Physical Status Classification (ASA PS) Levels. Ann Surg. 2006;243:636-44. https://doi.org/10.1097/01.sla.0000216508.95556.cc.

6. Gupta PK, Gupta H, Sundaram A, Kaushik M, Fang X, Miller WJ, et al. Development and Validation of a Risk Calculator for Prediction of Cardiac Risk After Surgery. Circulation. 2011;124:381-7. https://doi.org/10.1161/circulationaha.110.015701.

7. Protopapa KL, Simpson JC, Smith NCE, Moonesinghe SR. Development and validation of the Surgical Outcome Risk Tool (SORT). Br J Surg. 2014;101:1774-83. https://doi.org/10.1002/bjs.9638.

8. Chan DXH, Sim YE, Chan YH, Poopalalingam R, Abdullah HR. Development of the Combined Assessment of Risk Encountered in Surgery (CARES) surgical risk calculator for prediction of postsurgical mortality and need for intensive care unit admission risk: a single-center retrospective study. BMJ Open. 2018;8:e019427. https://doi.org/10.1136/bmjopen-2017-019427.

9. Chiew CJ, Liu N, Wong TH, Sim YE, Abdullah HR. Utilizing Machine Learning Methods for Preoperative Prediction of Postsurgical Mortality and Intensive Care Unit Admission. Ann Surg. 2020;272:1133-9. https://doi.org/10.1097/SLA.0000000000003297.

10. Helkin A, Jain SV, Gruessner A, Fleming M, Kohman L, Costanza M, et al. Impact of ASA score misclassification on NSQIP predicted mortality: a retrospective analysis. Perioper Med (Lond). 2017;6:23. https://doi.org/10.1186/s13741-017-0076-1.

11. Vogt AW, Henson LC. Unindicated preoperative testing: ASA Physical status and financial implications. J Clin Anesth. 1997;9:437-41. https://doi.org/10.1016/s0952-8180(97)00060-3.

12. Owens WD, Felts JA, Spitznagel EL. ASA Physical Status Classifications. A Study of Consistency of Ratings. Anesthesiology. 1978;49:239-43.

13. Haynes SR, Lawler PGP. An assessment of the consistency of ASA physical status classification allocation. Anaesthesia. 1995;50:1959. https://doi.org/10.1111/j.1365-2044.1995.tb04554.x.

14. Mak PHK, Campbell RCH, Irwin MG. The ASA Physical Status Classification: Inter-observer Consistency. Anaesth Intensive Care. 2002;30:633-40. https://doi.org/10.1177/0310057x0203000516.

15. Cassai AD, De Cassai A, Boscolo A, Tonetti T, Ban I, Ori C. Assignment of ASA-physical status relates to anesthesiologists' experience: a survey-based national-study. Korean Journal of Anesthesiology. 2019;72:53-9. https://doi.org/10.4097/kja.d.18.00224.

16. Riley RH, Holman CDJ, Fletcher DR. Inter-Rater Reliability of the ASA Physical Status Classification in a Sample of Anaesthetists in Western Australia. Anaesth Intensive Care. 2014;42:614-8. https://doi.org/10.1177/0310057x1404200511.

17. Ranta S, Hynynen M, Tammisto T. A survey of the ASA physical status classification: significant variation in allocation among Finnish anaesthesiologists. Acta Anaesthesiol Scand. 1997;41:629-32. https://doi.org/10.1111/j.1399-6576.1997.tb04755.x.

18. Curatolo C, Goldberg A, Maerz D, Lin H-M, Shah H, Trinh M. ASA physical status assignment by non-anesthesia providers: Do surgeons consistently downgrade the ASA score preoperatively? J Clin Anesth. 2017;38:123-8. https://doi.org/10.1016/j.jclinane.2017.02.002.

19. Tollinche LE, Yang G, Tan K-S, Borchardt R. Interrater variability in ASA physical status assignment: an analysis in the pediatric cancer setting. J Anesth. 2018;32:211-8. https://doi.org/10.1007/s00540-018-2463-2.

20. Burgoyne LL, Smeltzer MP, Pereiras LA, Norris AL, de Armendi AJ. How well do pediatric anesthesiologists agree when assigning ASA physical status classifications to their patients? Paediatr Anaesth. 2007;17:956-62. https://doi.org/10.1111/j.1460-9592.2007.02274.x.

21. Goldman L, Caldera DL, Nussbaum SR, Southwick FS, Krogstad D, Murray B, et al. Multifactorial Index of Cardiac Risk in Noncardiac Surgical Procedures. N Engl J Med. 1977;297:845-50. https://doi.org/10.1056/nejm197710202971601.

22. Landis JR, Koch GG. The measurement of observer agreement for categorical data. Biometrics. 1977;33:159-74.

Page $10 / 12$ 
23. DeLong ER, DeLong DM, Clarke-Pearson DL. Comparing the areas under two or more correlated receiver operating characteristic curves: a nonparametric approach. Biometrics. 1988;44:837-45.

24. Kuza CM, Hatzakis G, Nahmias JT. The Assignment of American Society of Anesthesiologists Physical Status Classification for Adult Polytrauma Patients: Results From a Survey and Future Considerations. Anesth Analg. 2017;125:1960-6.

https://doi.org/10.1213/ANE.0000000000002450.

25. Knuf KM, Maani CV, Cummings AK. Clinical agreement in the American Society of Anesthesiologists physical status classification. Perioper Med (Lond). 2018;7:14. https://doi.org/10.1186/s13741-018-0094-7.

26. Sankar A, Johnson SR, Beattie WS, Tait G, Wijeysundera DN. Reliability of the American Society of Anesthesiologists Physical Status Scale in Clinical Practice. Br J Anaesth. 2014;113:424-32. https://doi.org/10.1093/bja/aeu423.

27. Abouleish AE, Vinta SR, Shabot SM, Patel NV, Hurwitz EE, Krishnamurthy P, et al. Improving agreement of ASA physical status class between pre-anesthesia screening and day of surgery by adding institutional-specific and ASA-approved examples: a quality improvement project. Perioper Med (Lond). 2020;9:34. https://doi.org/10.1186/s13741-020-00162-4.

28. Routine preoperative tests for elective surgery. National Institute for Health and Care Excellence (NICE). Guidance 2016. https://www.nice.org.uk/guidance/ng45 (accessed April 2021).

29. Hurwitz EE, Simon M, Vinta SR, Zehm CF, Shabot SM, Minhajuddin A, et al. Adding Examples to the ASA-Physical Status Classification Improves Correct Assignment to Patients. Anesthesiology. 2017;126:614-22. https://doi.org/10.1097/ALN.0000000000001541.

30. Nie X, Mattke S, Predmore Z, Liu H. Upcoding and Anesthesia Risk in Outpatient Gastrointestinal Endoscopy Procedures. JAMA Intern Med. 2016;176:855-6. https://doi.org/10.1001/jamainternmed.2016.1244.

\section{Figures}

Patients > 18 years old presenting for surgery from 2017 to 2019 $(n=72071)$

$\longrightarrow \begin{gathered}\text { Exclude patients not explicitly coded as elective surgeries } \\ (\mathrm{n}=22091)^{*}\end{gathered}$

$\longrightarrow \begin{gathered}\text { Exclude patients scored as ASA } 5 \text { or } 6 \text { by either surgeon or } \\ \text { anesthesiologist }(n=51)^{*}\end{gathered}$

Patients after only elective surgeries retained $(n=49963)$

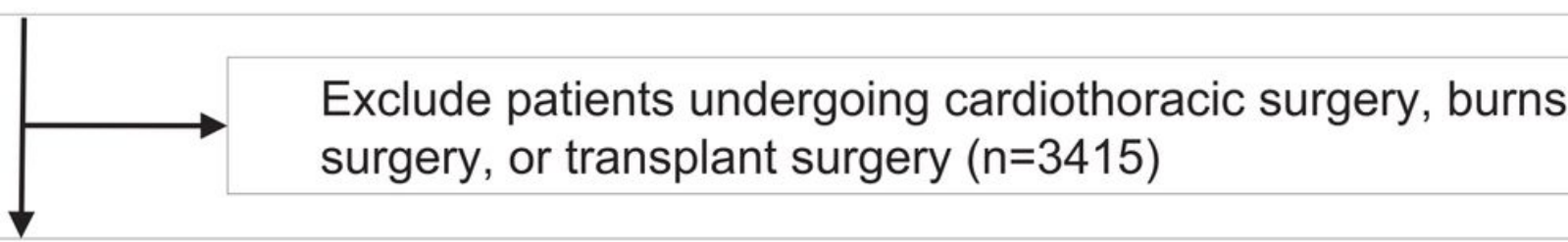

Patients not undergoing cardiothoracic surgery, burns surgery, or transplant surgery $(n=46548)$

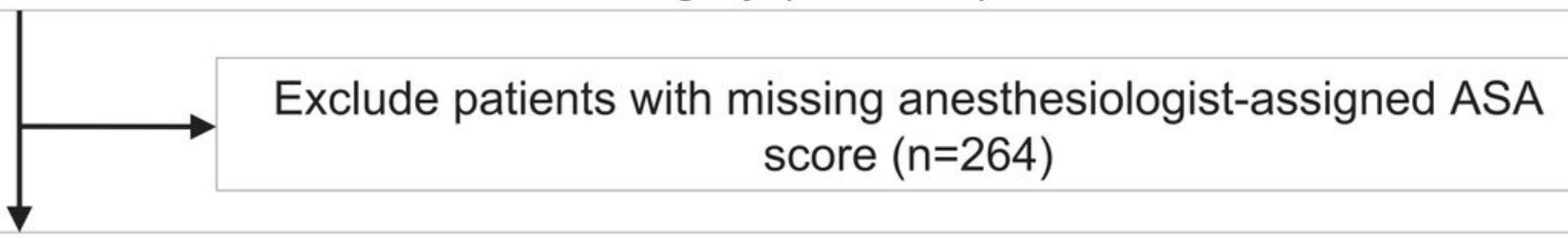

Patients with valid surgeon and anesthesiologist ASA score $(n=46284)$ 


\section{Figure 1}

Study flow diagram for patient cohort definition *The exclusions for patients not explicitly coded as elective surgeries and patients scored ASA 5 or 6 are overlapping categories, and as a result sum to more than the difference between the first two steps

\section{Figure 2}

Odds Ratio Plots for Risk of Adverse Outcomes with Different Levels of ASA Discordance (2A) Odds Ratio for death within 30 days; (2B) Odds Ratio for death within 1 year; (2C) Odds Ratio for ICU admission > 24 hours. A lower surgeon ASA score as compared to the anesthesiologist score was associated with all three outcomes. On the other hand, a lower anesthesiologist ASA score was only associated with ICU admission $>24$ hours but not death at 30 days or 1 year.

\section{Figure 3}

Composite plot of AUCs for prediction of adverse outcomes using surgeon and anesthesiologist ASA scores (3A) AUC of 30-day mortality for anesthesiologist and surgeon-assigned ASA scores (3B) AUC of 1-year mortality for anesthesiologist and surgeon-assigned ASA scores (3C) AUC of ICU admission $>24$ hours for anesthesiologist and surgeon-assigned ASA scores

\section{Supplementary Files}

This is a list of supplementary files associated with this preprint. Click to download.

- Additionalfile1.docx

- Additionalfile2.docx 